\title{
Uma Análise Comparativa entre Repositórios de Recursos Educacionais Abertos para a Educação Básica
}

\author{
Rodrigo Medeiros ${ }^{1}$, Marcos Doarte ${ }^{1}$, José Viterbo ${ }^{1}$, Cristiano Maciel $^{2}$, \\ Clodis Boscarioli ${ }^{3}$ \\ ${ }^{1}$ Universidade Federal Fluminense (UFF) - Niterói/RJ \\ ${ }^{2}$ Universidade Federal de Mato Grosso (UFMT) - Cuiabá/MT \\ ${ }^{3}$ Universidade Estadual do Oeste do Paraná (Unioeste) - Cascavel/PR \\ \{rodrigo_medeiros, mvdrocha, jviterbo\}@id.uff.br, cmaciel@ufmt.br \\ clodis.boscarioli@unioeste.br
}

\begin{abstract}
Open Educational Resources (OER) aim to make knowledge freer and more accessible to everyone. It is essential to provide repositories that promote the discovery, use and reuse of OERs so that they can be better used. Therefore, it is relevant to assess whether, in Brazil, the OER Repositories (OERR) have the quality to support Basic Education. In this article, we develop a comparative analysis between Brazilian OERR focused on this segment. A number of repositories were selected based on the following criteria: being national, comprehensive and having contents focused exclusively or mainly on basic education. The selected repositories were evaluated using Quality Indicators. Despite the large number of OERR available, the survey revealed a low number of OERR aimed at basic education, especially those with high rates on the criteria and indicators used, which demonstrates that there is still a way to go in this area.
\end{abstract}

Resumo. Os Recursos Educacionais Abertos (REA) visam tornar o conhecimento mais livre e acessivel para todos. É fundamental disponibilizar repositórios que promovem a descoberta, o uso e a reutilização de REA para otimizar seu uso. É, portanto, relevante avaliar se, no Brasil, os Repositórios de REA (RREA) possuem qualidade para prestar apoio à Educação Básica. Neste artigo, realizamos uma análise comparativa entre RREA brasileiros voltados a esse segmento. Foram selecionados repositórios segundo os critérios: serem nacionais, abrangentes e terem conteúdos voltados exclusiva ou majoritariamente ao ensino básico. Os repositórios selecionados foram avaliados com o uso de Indicadores de Qualidade. Apesar do grande número de RREA disponíveis, a pesquisa revelou um baixo número de RREA voltados ao ensino básico, especialmente os com alto rendimento em relação aos critérios e indicadores utilizados, o que demonstra que ainda há um caminho a ser percorrido nessa área.

\section{Introdução}

O movimento em prol de Recursos Educacionais Abertos (REA), surgido em 2002 [UNESCO, 2002], está hoje inserido em um movimento bem mais amplo, chamado Educação Aberta (Open Education), que, segundo Inuzuka e Duarte [2012], “[...] 
é um movimento de pessoas e instituições que promovem ações com o objetivo tornar a educação mais livre e acessível para todos". Neste contexto, é possível verificar mudanças no curso das práticas educacionais, com o uso cada vez mais intensificado das tecnologias digitais. Neste campo, é desejável que haja uma relação de complementaridade entre os REA e o educador, permitindo o acesso a recursos que possibilitem a disseminação do conhecimento e a melhoria da aprendizagem. Na visão de Maciel e Backes [2012], "O uso de materiais didático-tecnológicos [...] à disposição do professor para reutilização em diversos contextos, dá mostra de que a parceria entre conteúdo, tecnologia e metodologia é bem-sucedida e, se incentivada, a tendência é aumentar o ganho no processo educacional".

Frente aos atuais desafios da interrupção da educação presencial devido à pandemia da COVID-19, os Recursos Educacionais Abertos ganharam ainda mais importância, tal que a UNESCO reformulou seu guia de uso de práticas para a Educação Aberta, uma vez que o antigo material não dava conta dos novos desafios enfrentados [Huang et al., 2020]. Seu objetivo foi o de contribuir para que governos e professores pudessem mitigar os efeitos negativos causados pela pandemia fazendo uso de Recursos Educacionais Abertos, reforçando sua importância.

Para que os REA possam ser acessados de forma gratuita e democrática, os repositórios possuem um papel fundamental [Borges et al., 2020]. Santos-Hermosa et al. [2017] chamaram a atenção para a importância da realização de estudos sobre até que ponto os repositórios de REA (RREA) existentes promovem a descoberta, o uso e a reutilização de recursos, a fim de ampliar a adoção e o impacto dos REA. Os autores destacaram ainda que uma das principais ações transformadoras propostas pela Comissão Europeia no documento Opening up Education seria melhorar a visibilidade dos REA de qualidade por meio do desenvolvimento de RREAs digitais abertos [Comissão-Europeia, 2013], incluindo aspectos educacionais e metadados para que os recursos mais relevantes sejam classificados e possam ser recuperados pelos professores e alunos, seus principais usuários.

Diante disso, é relevante questionar se, no Brasil, os RREA possuem capacidade de apoiar de forma satisfatória os processos de ensino e aprendizagem na Educação Básica. Assim, com o propósito de comparar os RREA, realizamos uma pesquisa de natureza aplicada, visando gerar resultados reprodutíveis, buscando, com abordagem exploratória, apontar os RREA nacionais que melhor apoiam professores e alunos do ensino básico brasileiro, além de servir como base para outras seleções possíveis. Baseamos os procedimentos técnicos em pesquisa bibliográfica e leitura de estudos relacionados ao tema. A análise se pautou em selecionar repositórios a partir dos seguintes critérios: serem nacionais; abrangentes - não se limitando a uma disciplina ou temática - e terem conteúdos voltados exclusiva ou majoritariamente à Educação Básica (Ensino Fundamental e Médio). Posteriormente, a partir do uso de Indicadores de Qualidade [Atenas and Havemann, 2014], em uma análise quantitativa, foram atribuídas pontuações aos repositórios selecionados.

Este documento segue assim organizado: Na Seção 2, apresentamos os Recursos Educacionais Abertos, abordando conceitos centrais para a compreensão do movimento REA. Na Seção 3, discutimos a metodologia proposta para a seleção e análise do corpus. Na Seção 4, apresentamos os resultados da seleção dos RREA. Na Seção 5, trazemos 
os resultados da análise comparativa entre os RREA selecionados. Por fim, na Seção 6, apresentamos nossas considerações finais e discutimos caminhos para pesquisas futuras.

\section{Recursos Educacionais Abertos e Repositórios}

Recursos Educacionais Abertos (REA) se instituem como materiais educacionais abertos, planejados e produzidos para atender o contexto educativo, e, em grande medida, disponíveis em repositórios educacionais [dos Anjos and Alonso, 2020]. O termo apareceu pela primeira vez em 2002 no Fórum da UNESCO que discutia os impactos dos Open Couserware (OCW) na educação superior de países em desenvolvimento [UNESCO, 2002]. A criação dos REA está intimamente ligada aos OCW, inicialmente uma iniciativa proposta do Massachusetts Institute of Tecnology (MIT), no ano de 2001, e são a expressão do ideal da instituição em relação à Educação a Distância naquele ano. REA e OCW fazem parte de um movimento maior chamado Educação Aberta que busca promover a democratização do ensino, partindo de preceitos calcados na pluralidade de contextos, de ideias e de recursos. Amiel aponta que a Educação Aberta "é uma tentativa de buscar alternativas sustentáveis para algumas das barreiras evidentes no que tange ao direito de uma educação de qualidade" [Amiel, 2012].

Mesmo não havendo um consenso em relação ao conceito de Educação Aberta, é possível elencar características comuns entre as diversas correntes de pensamento: 1. liberdade de espaço (casa, polos de aprendizagem, escola, entre outras possibilidades); 2. liberdade em relação à metodologia de ensino - se a instrução ocorrerá por módulos, créditos, entre outras; 3 . possibilidade de autoinstrução sem que isto a caracterize diferente do ensino formal, inclusive em relação à certificação; 4. gratuidade; 5. isenção de provas e outras formas de seleção para acesso à instrução ofertada; 6 . acessibilidade a pessoas portadoras de necessidades especiais ou em razão de desigualdades sociais; 7. utilização prioritária de REA [Rossi et al., 2012].

Boll et al. [2018] definem REA como:

[...] objetos, ferramentas, materiais ou técnicas de ensino e pesquisa, suportados por uma mídia, em domínio público ou com licença aberta, que permitem a cópia, o compartilhamento, a modificação e a distribuição, sem infringir os direitos autorais e com respeito à condição imposta pelo autor.

As autoras citam também objetos de aprendizagem, objetos educacionais, conteúdos digitais ou conteúdos abertos como exemplos de materiais REA. Indo ao encontro destas ideias, a visão expressa na Declaração da Cidade do Cabo (2007) coloca que o movimento REA:

...é constituído na crença de que todos devemos ter liberdade de utilizar, personalizar, melhorar e redistribuir os recursos educacionais sem restrições. Educadores, aprendizes e outros que compartilham dessa crença estão se unindo como parte de um esforço global para fazer da educação algo acessível e efetivo.

Desta forma, o ciclo de vida dos REA, representado pelos 4R (Review, Reuse, Remix e Redistribute - em português: Usar; Aprimorar; Recombinar; Distribuir) já aparecem bem delineados nesse documento. Vale destacar que atualmente foi incluído um novo "R"ao ciclo dos REA, o retain (manter, em português) [Hilu et al., 2015]. 
Há questões fundamentais para que um recurso educacional seja considerado aberto, quais sejam: permitir uso e reuso; re-propósito e modificação dos recursos; seu uso deve ser gratuito; deve garantir um maior acesso aos recursos didáticos e abranger todos os tipos de mídia digital, preferencialmente através de formatos abertos. Além disso, é importante que seu uso se paute na colaboratividade, estimulando a solidariedade intelectual e o enriquecimento do conhecimento.

Em relação à categorização dos REA, Camillieri, Ehlers e Pawlowski [2014] os subdividem, de forma mais ampla, em três categorias: 1. conteúdos de aprendizagem, que são o material em si; 2 . ferramentas, definidas como "softwares para apoiar o desenvolvimento, uso, reutilização e entrega de conteúdo de aprendizagem", e 3. recursos de implementação, que são as licenças utilizadas em recursos abertos. Diminuindo a granularidade classificatória dos tipos, os autores delineiam três categorias: criados por colaboradores (user-generated) versus criados por organizações (organisationally-produced resources); produzidos por pares (peer-produced) versus produzidos por iniciativa individual (individually-authored resources) e único (single) versus agregado (packaged OER).

Os conteúdos gerados por colaboradores são os mais abundantes na internet. Estes recursos são criados por qualquer pessoa e podem ou não possuir fins educacionais. De maneira geral, apresentam qualidade inconstante, tendo em vista que os criadores podem ter maior ou menor grau de expertise sobre o tema. Já os REA criados por organizações, também conhecidos como grandes recursos, são gerados a partir de projetos de Educação Aberta com fins estritamente educacionais e possuem objetivos e metodologia explícitas.

O uso de licenças abertas é um dos elementos mais importantes para o movimento dos REA, promovendo e encorajando o seu reuso e o compartilhamento. A licença de uso é um dos aspectos que define um recurso como sendo um Recurso Educacional Aberto, tendo em vista a liberdade de utilização por terceiros. Essa premissa indica que o material deve estar disponível sem que haja qualquer tipo de cobrança monetária, sendo sua obtenção, acesso, consumo, compartilhamento, ou mesmo adaptação, sem a necessidade prévia de autorização do proprietário. Para Butcher, Kanwar e Stamenka [2011]:

...o principal diferencial entre um REA e qualquer outro recurso educacional é sua licença. Assim, um REA é simplesmente um recurso educacional que incorpora uma licença que facilita a reutilização - e, potencialmente, a adaptação - sem primeiro solicitar permissão do detentor dos direitos autorais.

Os repositórios e buscadores de recursos digitais surgiram conjuntamente ao avanço da tecnologia, quando mudamos a forma de armazenar informações, transformando grande parte do que antes estava impresso em papel em um documento de texto online e fotos em mídias digitais. Para Maciel e Backes, "Um repositório funciona como uma biblioteca [...] de todas áreas do conhecimento". Os autores completam que "o principal objetivo dos repositórios é armazenar e disponibilizar os materiais construídos nas instituições acadêmicas ou governamentais ligadas à educação" [Maciel and Backes, 2012].

Como forma de solução para localizar os REA diante da grande disseminação dos repositórios, tem-se desenvolvido ferramentas específicas para a busca dos REA. Assim, viabilizaram-se condições de acessibilidade ao diminuir o esforço necessário para encontrar materiais de qualidade, pois oferecem operadores que permitem delimitar a consulta 
por meio de parâmetros comuns de buscadores conhecidos, como: palavra-chave, formato, idioma, sites e tamanho, além de critérios específicos, como tipos de uso do material e combinações de licenças.

Os repositórios que se encarregam em realizar o armazenamento e a distribuição de REA, são chamados Repositórios de Recursos Educacionais Abertos (RREA). Segundo Atenas e Havemann, podemos definir os RREA como "[...] plataformas que hospedam e facilitam o acesso destes recursos" [Atenas and Havemann, 2014], de maneira a “[...] apoiar educadores na busca de conteúdo, compartilhando seus próprios recursos, reutilizando e avaliando materiais e adaptando materiais feitos por ou em colaboração com outros membros da comunidade" [Atenas and Havemann, 2014].

Diante desta dificuldade, surgem, por meio do movimento da Educação Aberta e do avanço das tecnologias diversas, iniciativas mais pontuais - como o uso de blogs, fóruns, redes sociais, wikis, e-mail e canais e vídeos disponibilizados no YouTube - que muitas vezes aproveitam a estrutura das ferramentas existentes para o compartilhamento de materiais abertos. Por se tratarem de projetos menores, geralmente se propõem a difundir conteúdo com uma temática especializada, o que fomentou os investimentos em RREA, especialmente órgãos educacionais estatais, como Universidades, de parcerias público-privadas ou mediante o terceiro setor. Outra possibilidade, é a contribuição direta de órgãos supra-nacionais, como a UNESCO, na criação e manutenção de RREA.

\section{Percurso Metodológico}

Essa pesquisa tem natureza aplicada e visa gerar resultados aplicáveis e reprodutíveis. O objetivo é apontar os RREA nacionais que melhor apoiam professores e alunos do ensino básico brasileiro. Nesta seção, relatamos a metodologia adotada para a avaliação dos RREA escolhidos. Inicialmente, apresentamos os critérios para a escolha dos RREA analisados. E, por fim, são descritos os critérios para a comparação destes repositórios e os elementos analíticos empregados para sua classificação.

\subsection{Critérios para a seleção dos RREA}

Vale inicialmente destacar que buscamos selecionar o maior número possível de Repositórios de Recursos Educacionais Abertos. Não obstante, se fez necessário estabelecer critérios para que a seleção nos retornasse RREA que se enquadrassem ao nosso objetivo.

O primeiro critério adotado foi que o RREA contivesse, se não apenas, ao menos majoritariamente, REA voltados à Educação Básica. Tal escolha se justifica pelo nível educacional deficitário do Brasil neste segmento, como pode ser verificado no último relatório de desempenho do PISA - Programa Internacional de Avaliação de Estudante -, principal avaliação da educação básica no mundo, realizado em 2018. Neste, o Brasil apareceu entre as 20 piores colocações no ranking.

Outro critério fundamental para a seleção do nosso corpus foi que os RREA fossem de iniciativa nacional, pelos seguintes motivos: o primeiro diz respeito à acessibilidade do conteúdo disponibilizado, pois incluir repositórios cuja interface e recursos estejam em outro idioma, seria restringir a poucos o acesso aos REA e, portanto, um contrassenso em relação ao que preconiza o movimento; o segundo, pelas especificidades do sistema educacional e da cultura brasileira, pois familiaridades nestes aspectos podem contribuir à facilitação de processos de aprendizagem. Definimos como último critério 
para a seleção dos RREA que os mesmos contemplassem diversos tipos de saberes relacionados à Educação Básica. Com isso, a intenção foi a de selecionar repositórios mais completos e que melhor atendessem às necessidades de educadores e estudantes brasileiros. Assim, foram descartados da seleção repositórios que se restringiam a apenas uma determinada temática ou área de conhecimento.

\subsection{Critérios para comparação dos RREA}

Comparar é sempre um processo delicado e crítico, pois caso os critérios não sejam definidos de forma transparente, corremos o risco de ser parciais e, com isto, deturpar a pesquisa científica e transformá-la em opiniões. Antes de definir qual seria a forma de classificar os RREA, realizamos uma análise de trabalhos relacionados ao tema.

A partir da revisão de literatura, verificamos a existência de poucos trabalhos que realizam análises acerca de Repositórios de Recursos Educacionais Abertos, especialmente em relação à verificação de qualidade. Não obstante, pontuamos alguns trabalhos que contribuíram para uma melhor compreensão do tema. Amiel e Soares apresentam "um método de auditoria para averiguar a "abertura" de repositórios de recursos abertos com enfoque nos termos de uso" [Amiel and Soares, 2016]. Esses autores realizam uma análise quantitativa de 50 RREA latino-americanos voltados à Educação Básica, buscando investigar se há informações referentes às licenças utilizadas e, quando estas estão presentes, se há consistência entre o descrito e a realidade.

Em um segundo momento, procedemos a análise de Zanin [2017], cuja temática é bastante próxima ao anterior, pois ambos verificam informações sobre as licenças em RREA. Por um lado, a pesquisa de Zanin se aproxima mais da nossa, uma vez que realiza uma análise de repositórios nacionais, mas por outro lado, o faz a partir de repositórios de todos os níveis de ensino. Ambos os trabalhos citados acima focam suas pesquisas apenas nas licenças de uso dos RREA.

A proposta deste artigo é proceder a uma análise comparativa e classificatória a partir de aspectos dos RREA, a fim de verificar qual é o melhor dentre os resultantes da busca realizada. Com este intuito, verificamos que a análise por Indicadores de Qualidade (IQ), presentes em Atenas e Havemann [2014], resultado de um esforço dos autores em compilar os principais elementos presentes em trabalhos sobre o tema, é a que melhor se adequa a uma comparação de repositórios, pois, diferentemente dos anteriores, estes autores se utilizaram de diversos aspectos avaliativos. Atenas e Havemann propuseram dez Indicadores de Qualidade para a análise de RREA. Em nossa pesquisa, porém, não consideramos o critério tradução, uma vez que a análise ocorreu apenas com RREA nacionais. Entretanto, incluímos o critério gamificação, uma estratégia bastante empregada para gerar engajamento de usuários [Neto and Júnior, 2017].

A seguir, são descritos os nove indicadores de qualidade de RREA segundo a perspectiva de Atenas e Havemann [2014], e o indicador gamificação, adotados.

- Recursos em Destaque (IQ1). O destaque na apresentação de REA a partir de critérios pré-determinados pode ser um importante aliado para educando e educadores para facilitar sua seleção.

- Ferramentas de Avaliação (IQ2). Esse fator gera maior confiança por parte dos usuários, além de colaborar para o engajamento do público-alvo. Desta forma, 
“confiar nos usuários para avaliar o conteúdo pode ajudar a ganhar uma massa crítica de usuários engajados em REA que apoiam o controle de qualidade dos recursos" [Atenas and Havemann, 2014].

- Revisão dos Pares (IQ3). O processo de revisão por pares consiste na verificação dos recursos submetidos por especialistas no assunto, a fim de prevenir a distribuição de materiais de baixa qualidade ou mesmo de materiais com intuito negativo.

- Autoria dos Recursos (IQ4). Publicizar a autoria dos REA encoraja os usuários a compartilharem seus materiais, tendo confiança de que será reconhecida a sua propriedade intelectual, e, por outro, pode ser servir como desestímulo àqueles que porventura pensem em inserir um material que acreditam não ter boa qualidade.

- Palavras-Chave (IQ5). Tem o propósito de criar elementos extras, além das taxonomias padronizadas, que possam ajudar o usuário a encontrar e selecionar materiais que estejam mais condizentes com as suas necessidades.

- Uso de metadados padronizados (IQ6). O uso de metadados padronizados pode "funcionar em vários países, idiomas e culturas" [OCDE, 2007], além de contribuir para a interoperabilidade entre repositórios, sendo um importante elemento de alavancagem do reuso de REA. Na esfera educacional, os padrões de metadados mais utilizados são o Dublin Core, o SCORM e LOM (desenvolvido pela IEEE).

- Inclusão de Ferramentas de Mídia Social (IQ7). Uma vez que um dos principais elementos do movimento REA é a interação social, a integração com elementos de mídia social pode ser um valioso fator para gerar engajamento, ao criar "uma comunidade de usuários que colaborem, discutam, critiquem, usem, reutilizem" [Atenas and Havemann, 2014].

- Indicação do tipo de licença utilizada (IQ8). Licenças abertas são uma importante ferramenta para facilitar o uso, reuso, adaptação e compartilhamento de propriedade intelectual. As licenças específicas para o compartilhamento de REA devem estar expressas de forma clara, para resguardar os direitos de seus autores e usuários.

- Disponibilização do código-fonte ou dos arquivos originais (IQ9). É mais uma prática que está ancorada na premissa de compartilhamento do conhecimento. $\mathrm{O}$ próprio RREA pode ser considerado um Recurso Educacional Aberto, caso ele disponibilize seu código-fonte ou os arquivos originais editáveis.

- Gamificação (IQ10). Gamificação pode ser definido como o "uso de elementos de jogos em um contexto, produto ou serviço que não seja um jogo"[Neto and Júnior, 2017]. Por acreditarmos que a gamificação possa contribuir para elevar o engajamento de usuários, especialmente dos mais jovens, optamos por inseri-lo como um novo aspecto para a mensuração da qualidade de RREA.

\subsection{Valoração dos Indicadores de Qualidade}

Ao analisarmos os Repositórios de Recursos Educacionais Abertos, percebemos que, para muitos critérios, não era possível realizar uma avaliação binária do tipo: possui ou não possui. Desta forma, nos baseamos no framework LORI (Learning Object Review Instrument) [Nesbit et al., 2009], utilizado para a avaliação Objetos de Aprendizagem, que classifica objetos educacionais de acordo com critérios pré-estabelecidos. Entretanto, como 
os critérios avaliados pelo LORI possuem aspectos mais fortemente qualitativos do que os que definimos, com uma escala de valores indo de 1 a 5 [Nesbit et al., 2009], tal que adaptamos o método atribuindo uma valoração de 0 a 2 para cada critério definido, sendo 0 : não possui o elemento; 1: possui parcialmente e 2: possui.

\section{Seleção dos RREA}

A busca pelos repositórios ocorreu a partir da consulta em três diferentes fontes, sendo duas delas buscadores de RREA — o Mapa de Iniciativa de Recursos Abertos (MIRA ${ }^{1}$ ) e o OpenDOAR ${ }^{2}$ - e a terceira foi o site Wikiversity ${ }^{3}$, cujo foco está na Educação. De acordo com os critérios discutidos na Subseção 3.1, separamos os repositórios sugeridos pelos buscadores nas seguintes categorias:

- CAT1 - não possui somente ou majoritariamente material de nível básico;

- CAT2 - não possui nacionalidade brasileira;

- CAT3 - possui material especializado em determinado assunto ou disciplina;

- CAT4 - apresenta problemas técnicos ou serviço descontinuado;

- CAT5 - satisfaz os critérios predefinidos.

Na Tabela 1, é possível visualizar o número total de repositórios retornados por categoria. Apenas oito repositórios se enquadraram na CAT5, ou seja, satisfizeram completamente os requisitos definidos. Vale destacar que, de todos os 8 (oito) RREA selecionados, somente um - Portal do Professor - é sugerido exclusivamente pelo Wikiversity, todos os outros aparecem nos resultados tanto deste site, quanto do MIRA. Nenhum repositório válido foi encontrado na pesquisa realizada no OpenDOAR.

Tabela 1. Resultado da busca por RREA

\begin{tabular}{c|c|c|c}
\hline Categorias & MIRA & OpenDOAR & Wikiversity \\
\hline CAT1 & 0 & 108 & 17 \\
CAT2 & 0 & 0 & 2 \\
CAT3 & 3 & 21 & 11 \\
CAT4 & 2 & 22 & 8 \\
CAT5 & 7 & 0 & 8
\end{tabular}

A seguir descrevemos cada um dos RREA selecionados, ordenados alfabeticamente, a fim de proporcionar uma visão geral de cada repositório válido na busca:

- Currículo Digital. Projeto da cidade de São Paulo, originado da colaboração de mais de 59 (cinquenta e nove) mil alunos e professores no decorrer do ano de 2017, fruto do Pátio Digital, ação da Secretaria Municipal de Educação com parceria da UNESCO, a qual busca a ampla abertura e difusão de materiais e dados públicos sobre a educação.

- Dia a Dia Educação. Ferramenta integrada à Secretaria de Estado da Educação do Paraná, lançada em 2004 e reestruturada em 2011, com o intuito de disponibilizar serviços, informações e REA para toda a comunidade escolar.

\footnotetext{
${ }^{1}$ https://mira.org.br

${ }^{2}$ https://v2.sherpa.ac.uk/opendoar

${ }^{3}$ https://pt.wikiversity.org/wiki
} 
- Educopédia. Desenvolvido pela Secretaria de Educação do Rio de Janeiro, em 2010, com a colaboração de cerca de 300 (trezentos) professores da rede municipal para formar o material pedagógico, que inclui itens relativos aos anos escolares e às categorias: Educação Infantil; Educação de Jovens e Adultos; Educação Especial e cursos para professores.

- Escola Digital. Criado em 2013 por iniciativa de fundações ligadas ao setor privado, por meio de parcerias com o setor público, foi implementado em mais de 30 (trinta) Secretarias Públicas de Educação. Trata-se de uma rede colaborativa, e cada vez que uma instância é criada, seu acervo passa a incorporá-la.

- Plataforma Anísio Teixeira. Iniciativa de 2008 do governo do Estado da Bahia, por meio do Instituto Anísio Teixeira, baseia-se no compartilhamento de REA voltados à Educação Básica e para a Formação Continuada dos Profissionais da Educação Básica das redes públicas, tendo atingido mais de cinco milhões de acessos, dentre os mais de dezessete mil recursos disponibilizados.

- Plataforma Integrada MEC. Surgiu por meio da iniciativa do Ministério da Educação no ano de 2015, com a proposta de reunir e disponibilizar REA em um único lugar, através de parcerias com os principais portais do Brasil.

- Portal do Professor. Surgiu em 2008 da parceria entre as Secretarias Estaduais e Municipais de Educação com o Ministério da Ciência e Tecnologia, e busca apoiar o processo de formação de professores brasileiros.

- REA Dante. Foi criado em 2011, para a disponibilização de REA produzidos principalmente pela equipe interna do Colégio Dante. O espaço incentiva a colaboração e o compartilhamento de ideias para melhorar a produção já existente e para permitir acesso amplo a conteúdo de qualidade.

\section{Análise Comparativa}

A análise dos repositórios foi realizada de forma imparcial, seguindo a estrutura de Indicadores de Qualidade previamente especificados. As notas foram atribuídas por dois dos cinco pesquisadores autores, tendo, contudo, os resultados sido revisados e avalizados pelos demais. Na Tabela 2 apresentamos todas as pontuações atribuídas aos RREA.

Tabela 2. Resultado da avaliação a partir dos Indicadores de Qualidade (IQ)

\begin{tabular}{|l|c|c|c|c|c|c|c|c|c|c|c|}
\hline RREA & IQ1 & IQ2 & IQ3 & IQ4 & IQ5 & IQ6 & IQ7 & IQ8 & IQ9 & IQ10 & Total \\
\hline Currículo Digital & 1 & 0 & 2 & 0 & 0 & 2 & 0 & 2 & 1 & 0 & 8 \\
\hline Dia a Dia Educação & 1 & 2 & 2 & 1 & 2 & 1 & 2 & 0 & 0 & 0 & 11 \\
\hline Educopédia & 0 & 0 & 2 & 0 & 0 & 1 & 0 & 2 & 0 & 0 & 5 \\
\hline Escola Digital & 2 & 2 & 2 & 2 & 2 & 2 & 2 & 2 & 0 & 0 & 16 \\
\hline Plat. Anísio Teixeira & 2 & 0 & 2 & 1 & 2 & 2 & 0 & 2 & 0 & 2 & 13 \\
\hline Plat. Integrada MEC & 2 & 2 & 2 & 2 & 2 & 2 & 2 & 2 & 2 & 1 & 19 \\
\hline Portal do Professor & 1 & 2 & 2 & 2 & 2 & 1 & 1 & 0 & 1 & 0 & 12 \\
\hline REA Dante & 0 & 0 & 2 & 0 & 0 & 2 & 0 & 2 & 0 & 0 & 6 \\
\hline
\end{tabular}

A fim de apresentar uma visão holística em relação aos RREA examinados, os agrupamos em três níveis de acordo com a pontuação obtida nos IQ, a saber: baixo rendimento, entre 0 e 9 pontos; médio rendimento, entre 10 e 15 pontos e alto rendimento, entre 16 e 20 pontos. Tanto a Plataforma Integrada MEC, quanto a Escola Digital apresentaram um alto rendimento em relação aos IQ estabelecidos. A primeira obteve o maior 
destaque, alcançando quase a pontuação máxima sendo, portanto, a mais completa entre todas as analisadas. Um ponto de grande destaque é o fato de possuir seu código aberto no repositório gitlab, o que estimula a melhoria contínua da ferramenta. Outro destaque foi a Escola Digital, que alcançou 16 (dezesseis) pontos dos 20 (vinte) possíveis. A plataforma não possui código-aberto, mas está presente em 30 Secretarias de Educação (municipais e estaduais) no Brasil por meio de parcerias estabelecidas, contudo, não fica claro se isto se faz de maneira dispendiosa ou não.

Três plataformas se enquadraram na categoria médio rendimento: Plataforma Anísio Teixeira, o Portal do Professor e o Dia a Dia Educação. Podemos considerá-las ferramentas satisfatórias, mas que precisam ser aprimoradas em alguns aspectos, conforme demonstrado pelos IQ da Tabela 2. Já as plataformas que obtiveram os resultados menos satisfatórios ante aos critérios analisados, atingindo um baixo rendimento, foram: Educopédia, REA Dante e Currículo Digital de São Paulo, com, respectivamente, 5 (cinco), 6 (seis) e 8 (oito) pontos. Duas delas são iniciativa de Secretarias Municipais de Educação e uma da escola privada Dante Alighieri. Estas possuem restrições ao seu uso, especialmente em relação à submissão de material.

\section{Considerações Finais}

Verificamos, pela análise comparativa realizada, que mesmo havendo poucos Repositórios de Recursos Educacionais Abertos voltados à Educação Básica, existem plataformas altamente qualificadas capazes de cumprir de forma satisfatória a tarefa de apoiar os processos de ensino e aprendizagem nesses níveis educacionais e, mesmo aquelas que não atingiram alto rendimento, após alguns ajustes, podem cumprir este papel, o que demonstra que é importante reforçar o potencial existente para a evolução de iniciativas RREA voltadas à Educação Básica.

Vale destacar que, por conta do escopo desta pesquisa, alguns aspectos analíticos não foram contemplados, por exemplo, a não definição de quesitos qualitativos de análise. Análises qualitativas podem trazer resultados mais acurados quando buscamos definir critérios de qualidade de maneira mais específica, com vistas, por exemplo, a trazer o melhor resultado a um determinado perfil de usuário. Contudo, análises como a ora apresentada trazem importantes levantamentos, úteis a futuras pesquisas qualitativas.

Outras questões não contempladas dizem respeito à análise de requisitos não funcionais dos RREA e à análise de características ligadas à Interação Humano-Computador (IHC). Apesar de os Indicadores de Qualidade tangenciarem alguns aspectos de IHC, como a usabilidade, acreditamos ser ideal avaliar os repositórios também a partir dos conceitos consolidados dentro desta disciplina, uma vez que as realidades educacionais nacionais nem sempre dispõem de especialistas para auxiliarem o trabalho dos professores com tecnologias digitais, de forma que precisam ser acessíveis para serem adotadas por eles em suas intencionalidades pedagógicas e, também, compatíveis com os recursos de infraestrutura disponíveis nas escolas públicas, em especial, que podem ter defasagem de atualização de seus laboratórios de Informática.

Em pesquisas futuras, pretendemos avançar com as questões supracitadas, nos valendo de diversos aspectos analíticos - tanto os já definidos nesta pesquisa, quanto os aspectos não contemplados - com o objetivo de construir um framework para a avaliação de Repositórios de Recursos Educacionais Abertos. 


\section{Referências}

Amiel, T. (2012). Educação aberta: configurando ambientes, práticas e recursos educacionais. Salvador: Edufba; São Paulo: Casa de Cultura Digital.

Amiel, T. and Soares, T. C. (2016). Identifying tensions in the use of open licenses in OER repositories. International Review of Research in Open and Distributed Learning, 17(3):122-137.

Atenas, J. and Havemann, L. (2014). Questions of quality in repositories of open educational resources: a literature review. Research in Learning Technology, 22.

Boll, C., Ramos, W., and Real, L. (2018). Dicionário crítico de educação e tecnologias e de educação a distância. pages 51-74.

Borges, F. F., Teixeira, J. A., and Acedo, S. O. (2020). Uso de repositórios de recursos educacionais abertos nas práticas pedagógicas: uma revisão sistemática. Revista Latinoamericana de Tecnología Educativa-RELATEC, 19(2):115-133.

Butcher, N., Kanwar, A., and Uvalic-Trumbic, S. (2011). A Basic guide to open educational resources $(O E R)$. Paris: UNESCO; Vancouver : COL.

Camilleri, A. F., Ehlers, U. D., and Pawlowski, J. (2014). State of the art review of quality issues related to open educational resources (OER).

Comissão-Europeia (2013). Communication from the commission to the european parliament. opening up education: Innovative teaching and learning for all through new technologies and open educational resources. Technical report, European Commission.

da Cidade do Cabo, D. (2007). Declaração da cidade do cabo para educação aberta: Abrindo a promessa de recursos educativos abertos. https://www. capetowndeclaration.org/translations/ portuguese-translation. Acessado: 15/07/2020.

dos Anjos, R. A. V. and Alonso, K. M. (2020). Educação a distância e os recursos educacionais abertos: compreensões, possibilidades e perspectivas. In Maciel, C. and Viterbo, J., editors, Computação e Sociedade - Volume 3 - A Sociedade, chapter 19, pages 66-96. EdUFMT, Cuiabá.

Hilu, L., Torres, P. L., and Behrens, M. A. (2015). REA (recursos educacionais abertos) - conhecimentos e (des)conhecimentos. Revista e-Curriculum, 13(1):130-146.

Huang, R., D., L., Tlili, A., Knyazeva, S., Chang, T. W., Zhang, X., Burgos, D., Jemni, M., Zhang, M., Zhuang, R., and Holotescu, C. (2020). Guidance on open educational practices during school closures: Utilizing oer under covid-19 pandemic in line with unesco oer recommendation.

Inuzuka, M. A. and Duarte, R. T. (2012). Produção de REA apoiada por MOOC. Salvador: Edufba; São Paulo: Casa de Cultura Digital.

Maciel, C. and Backes, E. M. (2012). Objetos de aprendizagem, objetos educacionais, repositórios e critérios para a sua avaliação. Ambientes virtuais de aprendizagem, 12(1):161-198.

Nesbit, J., Belfer, K., and Leacock, T. (2009). Instrumento para a avaliação de objectos de aprendizagem (LORI): Manual do usuário. 
Neto, J. C. d. S. and Júnior, J. B. d. J. (2017). Ludificação em engenharia de software: Tornando o processo de desenvolvimento em uma empresa um jogo. Revista de Engenharia e Pesquisa Aplicada, 02(4).

OCDE (2007). Giving Knowledge for Free: The Emergence of Open Educational Resources. OCDE publishing.

Rossi, B., Rossini, C., and Pretto, N. D. L. (2012). Recursos Educacionais Abertos: práticas colaborativas e políticas públicas.

Santos-Hermosa, G., Ferran-Ferrer, N., and Abadal, E. (2017). Repositories of open educational resources: An assessment of reuse and educational aspects. International Review of Research in Open and Distributed Learning: IRRODL, 18(5):84-120.

UNESCO (2002). Forum on the impact of open courseware for higher education in developing countries-Final Report. Technical report, UNESCO.

Zanin, A. A. (2017). Recursos educacionais abertos e direitos autorais: análise de sítios educacionais brasileiros. Revista Brasileira de Educação, 22(71). 